\title{
Tecnologias que empregam fármacos antiparasitários para tratamento da doença Chagas
}

\section{Technologies using antiparasitic drugs to treat Chagas disease \\ Tecnologías que utilizan medicamentos antiparasitarios para tratar la enfermedad de Chagas}

Cleide Ane Barbosa da Cruz | cleideane.barbosa@bol.com.br

Universidade Federal de Sergipe (UFS), Programa de Pós-Graduação em Ciência da Propriedade Intelectual. São Cristóvão, SE, Brasil.

Amanda Luiza Soares Silva | amandalsoaressilva@gmail.com

Universidade Federal de Sergipe (UFS), Programa de Pós-Graduação em Ciência da Propriedade Intelectual. São Cristóvão, SE, Brasil.

Eurydice Maria Dantas de Alencar | euridicedantas@bol.com.br

Universidade Federal de Sergipe (UFS), Programa de Pós-Graduação em Ciência da Propriedade Intelectual. São Cristóvão, SE, Brasil.

Normandia de Jesus Brayner dos Santos | normajbs@hotmail.com

Universidade Federal de Sergipe (UFS), Programa de Pós-Graduação em Ciência da Propriedade Intelectual. São Cristóvão, SE, Brasil.

Jane de Jesus da Silveira Moreira|jane240370@yahoo.com

Universidade Federal de Sergipe (UFS), Programa de Pós-Graduação em Ciência da Propriedade Intelectual. São Cristóvão, SE, Brasil.

Ana Eleonora Almeida Paixão | aepaixao@gmail.com

Universidade Federal de Sergipe (UFS), Programa de Pós-Graduação em Ciência da Propriedade Intelectual. São Cristóvão, SE, Brasil.

Iracema Machado de Aragão Gomes | aragao.ufs@gmail.com

Universidade Federal de Sergipe (UFS), Programa de Pós-Graduação em Ciência da Propriedade Intelectual. São

Cristóvão, SE, Brasil.

\section{Resumo}

Com o aumento de diagnósticos da doença de Chagas, surge a necessidade de desenvolvimento de fármacos que tenham maior efeito contra os parasitas. O presente artigo objetiva apresentar um mapeamento tecnológico dos documentos de patentes relacionadas a tecnologias que empregam fármacos antiparasitários para tratamento da doença Chagas, por intermédio do banco de patentes do United States Patent and Trademark Office - USPTO. A primeira patente das 117 analisadas foi depositada nessa base em 1976. Os resultados demonstram que os estudos sobre o tratamento da doença de Chagas por meio de fármacos antiparasitários estão em desenvolvimento, focados na prevenção da doença, e são subsidiados em sua maioria por iniciativas particulares. Há pouco interesse da indústria farmacêutica no desenvolvimento de novos fármacos para tratamento e as universidades não têm incentivo para desenvolvimento de pesquisas sobre esse tema.

Palavras-chave: Doença de Chagas; Trypanossoma cruzi; Antiparasitários; Patentes; Medicamentos. 


\begin{abstract}
With increasing diagnostic Chagas disease, it is necessary to develop drugs that have a greater effect against parasites. This article aims to present a technological mapping of patent documents related to technologies employing antiparasitic drugs to treat Chagas disease, through the patent database United States Patent and Trademark Office - USPTO. 117 patents were analyzed, and the first patent was filed on that basis in 1976. The results show that studies of the treatment of Chagas disease through antiparasitic drugs are in development, focused on preventing the disease, and most of them are subsidized by private initiatives. There is little interest from the pharmaceutical industry to develop new drugs for treatment and universities have no incentive to develop research in this area.
\end{abstract}

Keywords: Chagas disease; Trypanosoma cruzi; Antiparasitic Agents; Patents; Medicines.

\title{
Resumen
}

Con el aumento del diagnóstico de la enfermedad de Chagas, hay una necesidad de desarrollar fármacos que tengan un mayor efecto contra parásitos. Este artículo tiene como objetivo presentar un mapeo tecnológico de documentos de patentes relacionadas con las tecnologías que emplean fármacos antiparasitarios para tratar la enfermedad de Chagas, a través del United States Patent and Trademark Office - USPTO. Se analizaron 117 patentes; la primera patente había sido presentada en esa base en 1976. Los resultados muestran que los estudios sobre el tratamiento de la enfermedad de Chagas a través de medicamentos antiparasitarios están en desarrollo, centrados en la prevención de la enfermedad, y la mayoría es subvencionada por iniciativas privadas. Hay poco interés por parte de la industria farmacéutica para desarrollar nuevos fármacos para el tratamiento y las universidades no tienen incentivos para desarrollar la investigación en esta área.

Palabras clave: La enfermedad de Chagas; Trypanosoma cruzi; Medicamentos antiparasitários; Patentes; Drogas.

\footnotetext{
Contribuição dos autores:

Concepção e desenho do estudo: Cleide Ane Barbosa da Cruz, Amanda Luiza Soares Silva, Normandia de Jesus Brayner dos Santos. Aquisição, análise ou interpretação dos dados: Cleide Ane Barbosa da Cruz, Eurydice Maria Dantas de Alencar, Normandia de Jesus Brayner dos Santos.

Redação do manuscrito: Cleide Ane Barbosa da Cruz, Amanda Luiza Soares Silva, Eurydice Maria Dantas de Alencar, Normandia de Jesus Brayner dos Santos.

Revisão crítica do conteúdo intelectual: Jane de Jesus da Silveira Moreira, Ana Eleonora Almeida Paixão, Iracema Machado de Aragão Gomes.

Análise estatística: Cleide Ane Barbosa da Cruz, Amanda Luiza Soares Silva.
}

Declaração de conflito de interesses: Os autores declaram não existir conflito de interesse referente à elaboração do artigo.

Fontes de financiamento: CAPES, FAPITEC

Considerações éticas: Uma vez que o artigo faz uma análise de patentes utilizando a base do USPTO não houve necessidade de passar pelo comitê de ética e pesquisa.

Agradecimento/Contribuições adicionais: A CAPES e a FAPITEC pelo apoio a pesquisa.

Histórico do artigo: Submetido: 23.jan.2016 | Aceito: 27.jan.2016 | Publicado: 30.mar.2016

Dados abertos: Os dados do estudo foram retirados de uma base de dados sobre patente pública.

Apresentação anterior: $O$ conteúdo do artigo não foi apresentado anteriormente em eventos ou em outras publicações.

Licença: CC BY-NC atribuição não comercial. Com essa licença é permitido acessar, baixar (download), copiar, imprimir, compartilhar, reutilizar e distribuir os artigos, desde que para uso não comercial e com a citação da fonte, conferindo os devidos créditos de autoria e menção à Reciis. Nesses casos, nenhuma permissão é necessária por parte dos autores ou dos editores 


\section{Introdução}

A Tripanossomíase americana ou doença de Chagas, como é popularmente conhecida, foi descoberta no ano de 1909, pelo cientista brasileiro Carlos Chagas ${ }^{1}$. O cientista foi o primeiro a reunir os aspectos clínicos dessa doença após isolar o parasita Tripanosoma cruzi, um protozoário hemoflagelado da família Trypanosomatidae, concluir todo o ciclo biológico da doença e identificar o vetor ${ }^{2}$.

Chagas é transmitida ao homem por insetos hematófagos, mais conhecidos como barbeiros, através da corrente sanguínea, pelo protozoário parasita unicelular Trypanossoma cruzi. A transmissão da doença é feita por via da pele, por transfusões, por transplantes, de forma congênita e ainda pela via oral através da ingestão de alimentos crus contaminados com as fezes desses insetos ${ }^{3}$.

Estima-se que 12 milhões de pessoas no mundo estejam infectadas, e que aproximadamente $30 \%$ dos pacientes chagásicos desenvolvam a cardiomiopatia, manifestação mais grave da doença ${ }^{4}$. No entanto, os dados epidemiológicos mostram ainda que, no início de 1980, o número estimado de infectados estava em torno de 16 a 18 milhões, tendo incidência anual de 300 mil casos. Em 2007, esses valores diminuíram para 8 milhões de infectados com 50 mil novos casos por ano $^{5-6}$.

Recentemente, a OMS (Organização Mundial da Saúde) e a OPAS (Organização Pan-americana de Saúde) mostraram visão otimista sobre a doença de Chagas, em virtude do sucesso obtido por meio das ações sistematizadas e bem-sucedidas de programas regionais de controle epidemiológico, que possibilitaram reduzir significativamente a transmissão com base na utilização de pesticidas?

No que diz respeito aos medicamentos para o tratamento da doença de Chagas, atualmente, há apenas um disponível no mercado, o benznidazol (BNZ). Outro medicamento comercialmente conhecido como Nifurtimox teve seu uso interrompido por apresentar efeitos secundários. O BNZ tem algumas restrições, como a baixa eficácia na fase crônica da doença, a alta taxa de abandono do tratamento devido aos efeitos colaterais causados pelo medicamento, a de apresentar longo período de tratamento (entre 30 a 60 dias) e a inexistência de formulação pediátrica, mas mesmo com esses fatores restritivos ainda é o fármaco indicado pelos médicos ${ }^{8-9}$.

Porém, recentemente novos derivados triazólicos (posaconazol, ravuconazol, TAK-187) foram testados e, como vêm apresentando resultados experimentais promissores tanto em animais como em seres humanos, poderão ser uma alternativa para o surgimento de novos medicamentos ${ }^{10}$. Por isso, o desenvolvimento de fármacos antiparasitários, ou seja, de medicamentos utilizados contra os parasitas dos seres humanos e animais, pode trazer novas formas de tratamento dessa doença.

Esses fármacos podem surgir através de experimentos com produtos naturais ou sintéticos que sejam similares a compostos com reconhecida atividade contra outras doenças ou através de alvos metabólicos específicos para um determinado parasita que se quer atingir ${ }^{11}$.

Por isso, utiliza-se o mapeamento tecnológico. Seu uso contribui nos processos de tomada de decisão referentes à pesquisa, desenvolvimento e inovação de fármacos antiparasitários ${ }^{12}$. Além disso, os estudos prospectivos possibilitam intervenções planejadas resultantes da identificação tanto de oportunidades quanto de necessidades ${ }^{13}$, favorecendo o desenvolvimento de pesquisas.

Neste contexto, o presente artigo objetiva apresentar um mapeamento tecnológico dos documentos de patentes relacionadas a tecnologias empregando fármacos antiparasitários para tratamento da doença de Chagas, através do banco de patentes do United States Patent and Trademark Office - USPTO. 


\section{Metodologia}

Para a pesquisa de tecnologia protegida ou descrita de documentos de patentes referente ao tratamento da doença de Chagas por meio de fármacos antiparasitários, elaborou-se uma estratégia de busca que combinou um conjunto de palavras-chave (Chagas AND disease, Chagas AND disease AND treatment AND prevention AND medicine, Trypanosoma AND cruzi AND treatment AND medicine; Chagas AND disease AND antiparasitic AND drugs AND treatment) no campo de busca por título e/ou resumo dos pedidos de patentes levantados.

Utilizou-se a base de patentes do United States Patent and Trademark Office (USPTO), que contém dados de patentes americanas. No caso dessa pesquisa foram analisadas patentes concedidas na base de 1976 até a atualidade. Os detalhamentos dos critérios de análise e da estratégia da busca são apresentados na Tabela 1.

Tabela 1 Critérios e estratégias de buscas

\begin{tabular}{ll}
\hline Tipo de monitoramento & Internacional \\
\hline Período & 1976 a 2014 \\
\hline & Evolução temporal das patentes \\
Busca & Perfil do depositante \\
& Inventores \\
& Classificação Internacional de Patentes (CIP) \\
\hline
\end{tabular}

Fonte: Autoria própria (2015)

\section{Resultados e discussões}

Ao realizar as buscas na base do USPTO, foram empregados estrategicamente os termos "doença de Chagas" com outros sinônimos, com o intuito de verificar o número de documentos sobre o tratamento da doença por meio de fármacos antiparasitários. As palavras-chave, mencionadas anteriormente, utilizadas nessa pesquisa são apresentadas na Tabela 2, bem como os respectivos números de documentos encontrados em cada combinação de termos pesquisados.

Tabela 2 - Estratégia de busca no USPTO

\begin{tabular}{llc}
\hline Palavras-chave & $\begin{array}{l}\text { Estratégia de } \\
\text { busca }\end{array}$ & $\begin{array}{l}\text { Número de } \\
\text { patentes }\end{array}$ \\
\hline Chagas AND disease & 1 & 1779 \\
\hline Chagas AND disease AND treatment AND prevention AND medicine & 2 \\
\hline Trypanosoma AND cruzi AND treatment AND medicine & 3 & 1278 \\
\hline
\end{tabular}


Observando a Tabela 2 é possível perceber que, por meio da junção de outras palavras, o estudo pode se tornar mais restrito e resultar em um número menor de patentes. Nessa pesquisa foram analisados os 117 documentos encontrados com a junção Chagas AND disease AND antiparasitic AND drugs AND treatment, com o intuito de verificar documentos relativos a tratamentos para controle e combate dessa doença.

A Figura 1 apresenta a evolução temporal das patentes analisadas. Cabe destacar que o primeiro depósito ocorreu em 1976; posteriormente, novos depósitos ocorreram a partir de 1988, mas a alavancagem ocorreu somente a partir de 1994. Os anos que obtiveram o maior número de depósitos foram 2007, 2010, 2011 com oito depósitos cada e 2009 com nove. Em 2014, houve uma queda, com apenas três patentes depositadas.

Mesmo que o médico Carlos Chagas tenha descoberto em fevereiro de 1909, após isolar o parasita Tripanosoma cruzi, um protozoário hemoflagelado da família Trypanosomatidae, e concluído todo o ciclo biológico da doença, chamada de doença de Chagas² $^{2}$, percebeu-se com a pesquisa que o primeiro depósito de patente relativo ao tratamento da doença surgiu apenas em 1976.

Com relação aos anos que obtiveram maior número de depósitos, cabe salientar que, neles, as pesquisas no Brasil se alavancaram por conta do aumento do número de casos da doença de Chagas aguda. Em 2007, foram confirmados 156 casos agudos dessa doença e em 2009 foram registrados 220 casos; já em 2010 esse número caiu para 131 casos confirmados e, em 2011, volta a ter uma leve elevação com 190 casos confirmados $^{14}$.

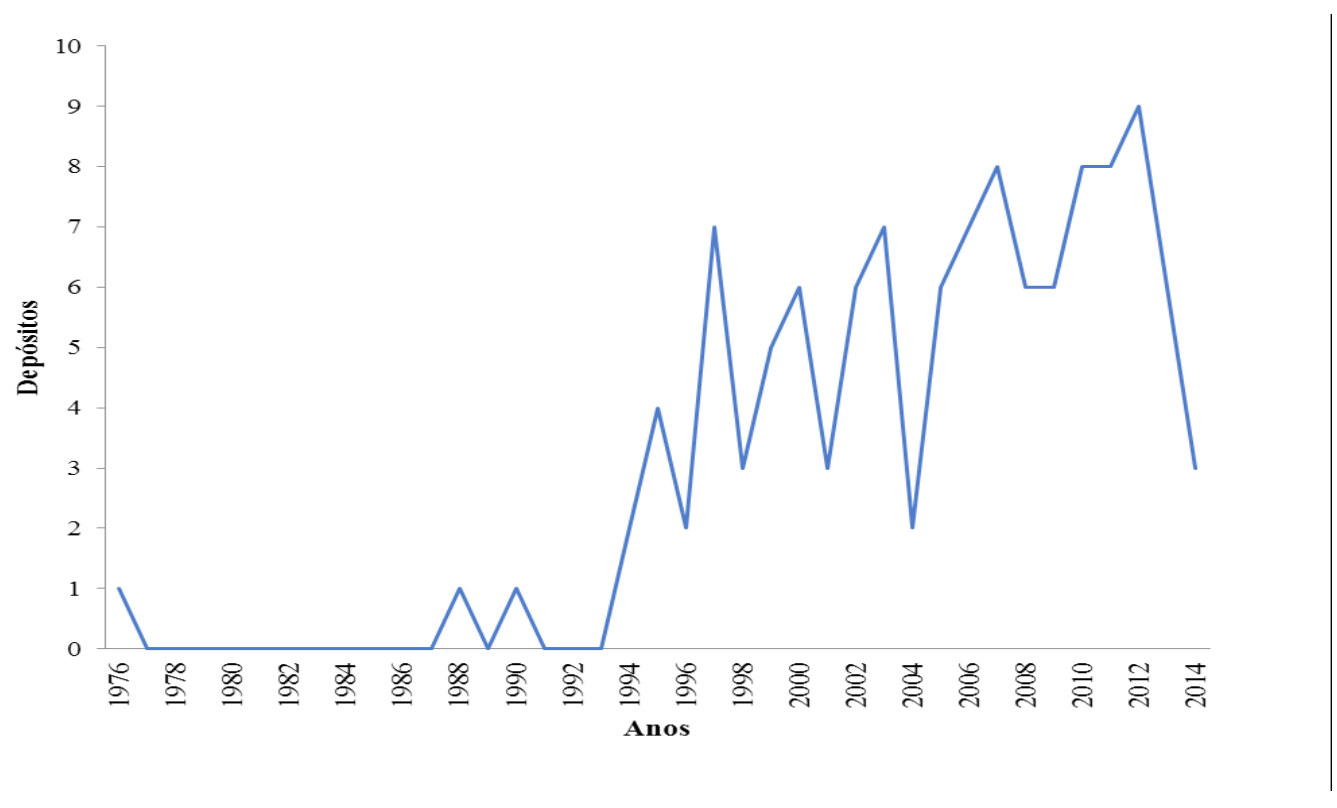

Figura 1 - Evolução anual do depósito de patentes de 1976 a 2014. Fonte: Autoria própria baseada em dados coletados no USPTO (2015).

Por meio da Figura 2, é possível observar que as patentes depositadas na base do USPTO estão relacionadas à seção A (necessidades humanas), com destaque para a classificação A61K 38/oo que foi a mais presente. Esta classificação refere-se a preparações medicinais contendo peptídeos e se distingue das demais apresentadas na pesquisa aqui analisada, por ser a que está mais presente nas patentes encontradas na base do USPTO.

É importante ressaltar que os peptídeos são biomoléculas que podem trazer um grande avanço na produção de novas drogas ${ }^{15}$. No caso aqui analisado, os peptídeos são novas alternativas para a indústria farmacêutica desenvolver medicamentos capazes de combater a doença de Chagas. 
Tabela 3 - Significado das classificações internacionais de patentes

\begin{tabular}{ll}
\hline Código de Classificação & Significado das classificações \\
\hline A61K 38/00 & Preparações medicinais contendo peptídeos \\
\hline A61K 31/4164 & Preparações medicinais contendo 1,3-Diazóis \\
\hline A61K 31/70 & $\begin{array}{l}\text { Preparações medicinais contendo carboidratos; açúcares; derivados dos } \\
\text { mesmos (sorbitol A61K 31/047) }\end{array}$ \\
\hline A61K 31/675 & $\begin{array}{l}\text { Preparações medicinais tendo nitrogênio como o heteroátomo de um } \\
\text { anel; por exemplo, fosfato piroxidal }\end{array}$ \\
\hline A61K 31/472 & $\begin{array}{l}\text { Preparações medicinais contendo isoquinolinas não-condensadas; por } \\
\text { exemplo, Papaverina }\end{array}$ \\
\hline A61K 31/44 & $\begin{array}{l}\text { Preparações medicinais piridinas não-condensadas; derivados } \\
\text { hidrogenados das mesmas }\end{array}$ \\
\hline A61K 31/35 & $\begin{array}{l}\text { Preparações medicinais tendo anéis de seis membros com um oxigênio } \\
\text { como o único heteroátomo de um anel }\end{array}$ \\
\hline A61K 31/136 & $\begin{array}{l}\text { Preparações medicinais tendo o grupo amino diretamente ligado ao anel } \\
\text { aromático; por exemplo, Benzenoamino }\end{array}$ \\
\hline A61K 31/00 & \begin{tabular}{l} 
Preparações medicinais contendo ingredientes ativos orgânicos \\
\hline A01N 63/00
\end{tabular} $\begin{array}{l}\text { Biocidas, repelentes ou atrativos de pestes ou reguladores do } \\
\text { crescimento de plantas contendo micro-organismos, vírus, fungos } \\
\text { microbiais, animais; por exemplo, nematóides ou substâncias produzidas } \\
\text { por ou obtidas de micro-organismos, vírus, fungos microbiais ou } \\
\text { animais; por exemplo, enzimas ou fermentados (contendo compostos de } \\
\text { constituição determinada A01N 27/00-A01N 59/00). }\end{array}$ \\
\hline
\end{tabular}

Fonte: Elaboração própria, a partir do INPI em 2015.

Uma patente encontrada com a classificação $\mathrm{A} 61 \mathrm{~K}$ 38/oo diz respeito a um método de prevenção ou inibição da infecção por um parasita ou vírus in vivo e foi depositada pelo Centre National de la Recherche Scientifique e pelo Instituto Gulbenkian de Ciência em 2006.

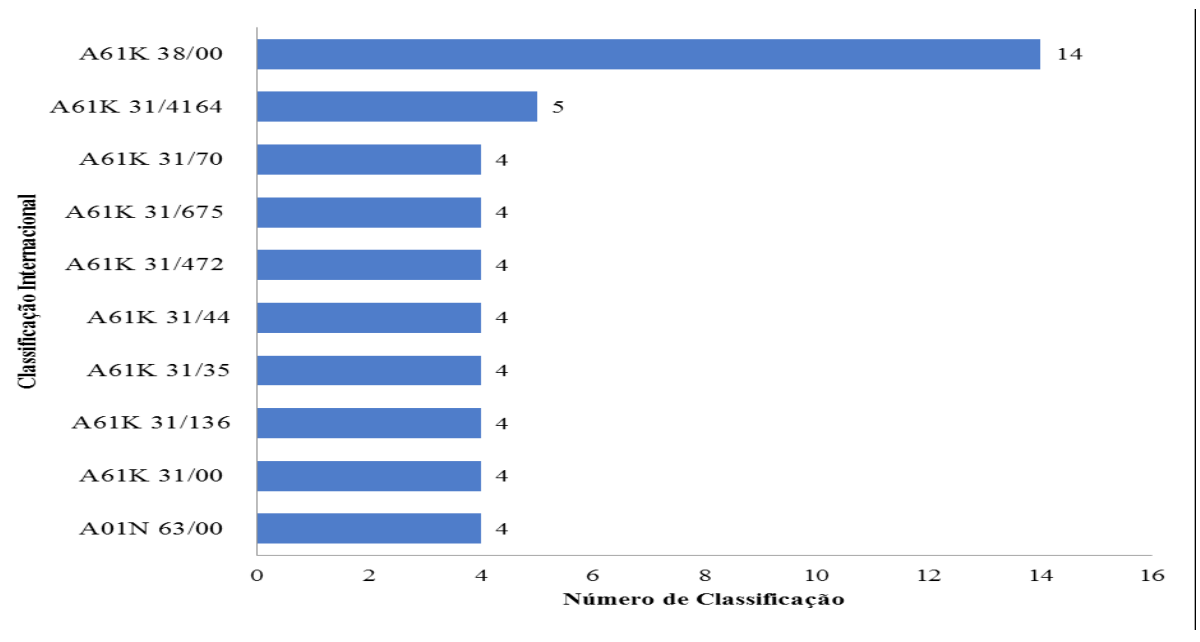

Figura 2 - Número de patentes por código de classificação internacional. Fonte: Autoria própria baseada em dados coletados no USPTO (2015). 
O perfil dos depositantes, destacado na Figura 3, indica que a maioria é constituída por inventores (64\%), 21\% são empresas e apenas 15\% são universidades. Esses dados mostram que ainda há necessidade de mais investimentos em universidades para o desenvolvimento de pesquisas e patentes relacionadas ao tratamento da doença das Chagas. Essa necessidade está ligada à falta de incentivo para parcerias entre universidades e empresas, que poderiam favorecer o desenvolvimento de novas pesquisas sobre doenças negligenciadas, como a de Chagas.

E mais, "as universidades e os centros de pesquisa são estruturas essenciais para o funcionamento de um sistema de inovação capaz de promover o desenvolvimento econômico de uma nação" ${ }^{16}$. Porém, para a universidade fazer parceria com o setor privado ainda assim precisa de incentivo, fiscal, financeiro e legal por parte de seu gestor maior, o governo federal ou estadual em casos poucos.

Na contramão da tendência observada foi encontrada uma patente depositada pela Fundação Oswaldo Cruz, relacionada aos compostos derivados de artesunato, processo de preparação, composição farmacêutica e utilização da respectiva medicina. A Fiocruz é uma instituição federal vinculada ao Ministério da Saúde, que busca desenvolver pesquisas para o tratamento das doenças tropicais no Brasil ${ }^{17}$.

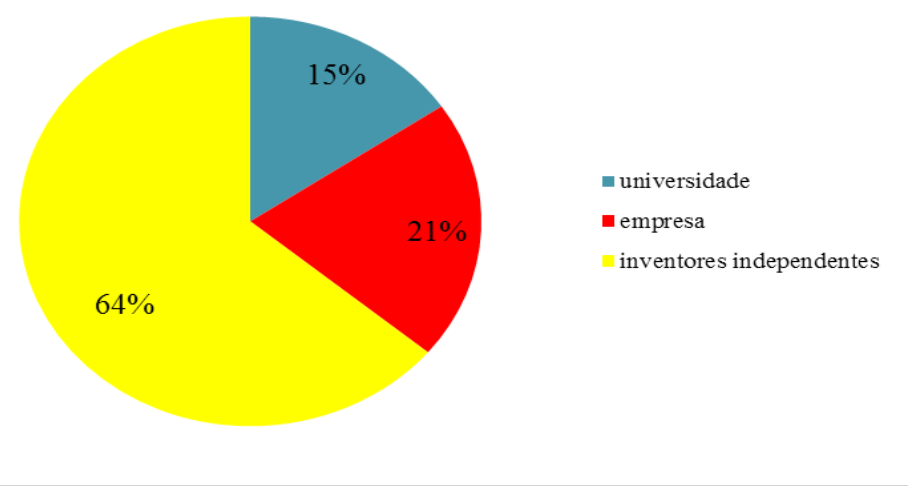

Figura 3 - Perfil dos depositantes

Fonte: Autoria própria baseada em dados coletados no USPTO (2015).

A Figura 4 destaca os inventores que realizaram mais de um depósito de patente. Entre os que possuem mais depósitos relacionados às palavras-chave utilizadas na base do USPTO, estão Dennis M. Schmatz, Sandra J. Rattray e Fred Ehrenkranz Cohen. Os demais inventores possuem 5 e 4 depósitos.

Dennis M. Schmatz atualmente realiza consultorias para empresas de biotecnologia, farmacêutica e organizações sem fins lucrativos. Sandra J. Rattray atua como vice-presidente da Johnson \& Johnson e Fred Ehrenkranz Cohen é o diretor da empresa de Autistica. Essas informações mostram, portanto, que os inventores destacados de acordo com os dados coletados na base do USPTO trabalham diretamente com empresas voltadas ao desenvolvimento de produtos farmacêuticos, embora não só de fármacos antiparasitários, mas também de produtos de higiene pessoal. 


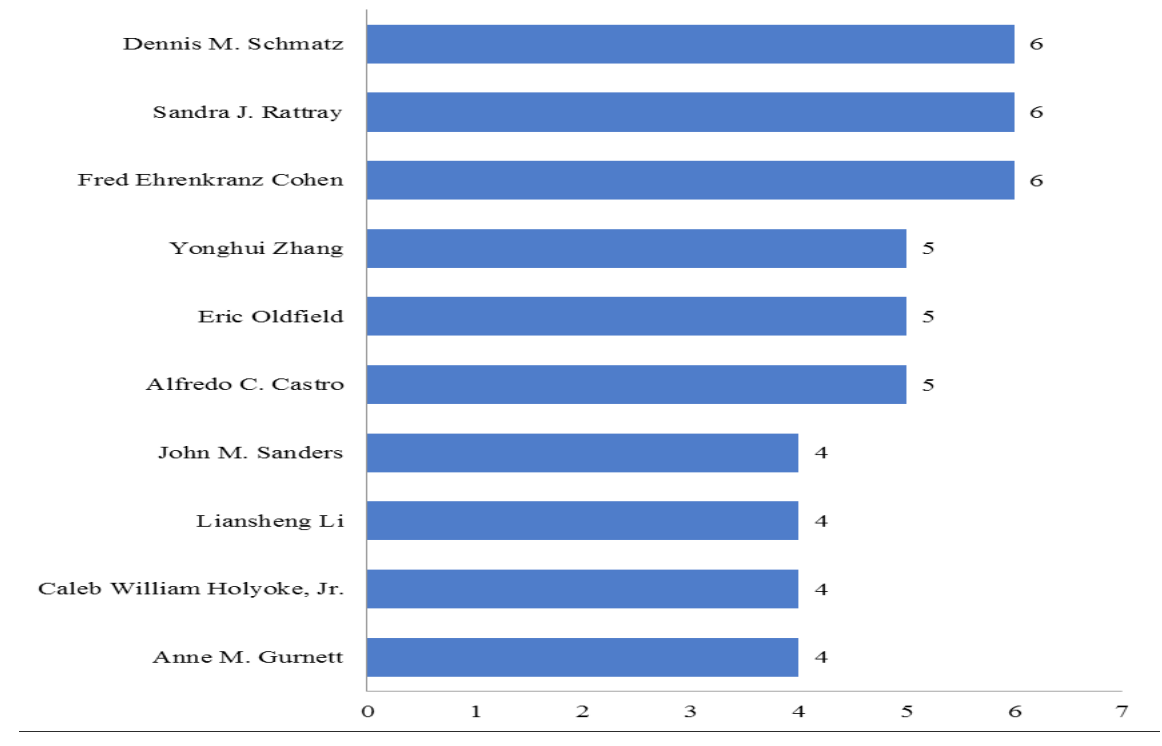

Figura 4 - Depósitos de patentes por inventores

Fonte: Autoria própria baseada em dados coletados no USPTO (2015).

Esses dados revelaram que há um número significativo de fármacos antiparasitários sendo desenvolvidos para o tratamento da doença de Chagas, mas que ainda há uma participação singela das universidades nesse campo de pesquisas.

\section{Conclusão}

A pesquisa na base de dados do USPTO sobre fármacos antiparasitários para o tratamento da doença de Chagas mostra que foram depositadas 117 patentes entre 1976 e 2014, sendo que 2009 foi o ano em que ocorreu o maior número de depósitos (nove).

Os dados denotam a falta de interesse da indústria farmacêutica e que a doença de Chagas é negligenciada embora tratável e curável. Trata-se de uma doença que acomete pessoas de baixa renda familiar ou na faixa da miséria, e com necessidade de tratamentos simples e eficazes.

Percebeu-se que a seção A (necessidades humanas) foi a única a aparecer nas patentes, relacionadas à subseção $\mathrm{A} 61 \mathrm{~K}$ e A01N que tratam de preparações medicinais e reguladores do crescimento de plantas micro-organismos, vírus, fungos microbiais, animais. Isso mostra que o foco das patentes relacionadas à doença encontra-se na prevenção através da regulação de plantas onde vivem os insetos e no tratamento com fármacos eficazes no combate à doença.

Assim, o desenvolvimento de fármacos antiparasitários poderão favorecer o surgimento de novas alternativas para tratamento da doença de Chagas, mas para isso também será necessário que se estimule parcerias entre universidades e empresas para que novas pesquisas sejam realizadas e novos medicamentos sejam criados. 


\section{Referências}

1. Galvão C, Justi SA. An overview on the ecology of Triatominae (Hemiptera: Reduviidae). Acta Tropica 2015; 151: 116-125.

2. Sobrinho JLS, Fontes DAF, Lyra MAM, Soares MFLR, Neto PJR. Doença de Chagas: cem anos de descoberta. Revista Brasileira de Farmácia 2009; 90: 283-289.

3. Fundação Oswaldo Cruz [Internet]. Doença de Chagas. 2013 - [citado 4 nov 2015]. Disponível em: http://portal.fiocruz.br/pt-br/content/historia

4. Buhaya MH, Galvan S, Maldonado RA. Incidence of Trypanosoma cruzi infection in triatomines collected atIndio Mountains Research Station. Acta Tropica 2015; 150: 97-99.

5. Senior, K. Chagas disease: moving towards global elimination. Lancet infect. Dis 2007; 7: 572.

6. Develoux M, Lescure FX, Loup GL, Pialoux G. Maladie de Chagas. Chagas disease. La Rev med int 2009; 30: $686-695$.

7. Noya BA, González ON. An ecological overview on the factors that drives to Trypanosoma cruzi oral transmission. Acta Tropica 2015; 151: 94-102.

8. Paula FR, Jorge SD, Almeida LV, Pasqualoto KFM, Tavares LC. Molecular modeling studies and in vitro bioactivity evaluation of a set of novel 5-nitro-heterocyclic derivatives as anti-T. cruzi agents. Bioorgan. Med. Chem 2009; 17: 2673-2679.

9. Mezencev R, Galizzi M, Kutschy P. Docampo, R. Trypanosoma Cruzi: antiproliferative effect of indole phytoalexins on intracellular amastigotes in vitro. Exp. Parasitol 2008; 122: 66-69.

10. Urbina JA. New Insights in Chagas'Disease Treatment. Drugs Fut 2010; 35: 409-410.

11. Izumi E, Morello LG, Ueda-Nakamura T, Yamada-Ogatta SF, Dias-Filho BP, Cortez DA, Ferreira IC, Morgado-Diaz JÁ, Nakamura CV. Tryapanosoma cruzi: antiprotozoal activity of parthenolide obtained from Tanacetum parthenium (L.) Schultz Bip. (Asteraceae, Compositae) against epimastigote and amastigote forms. Exp. Parasitol 2008; 118: 324-330.

12. Mayerhoff, ZDVL. Uma análise sobre os estudos de prospecção tecnológica. Cad de prospecção, n. 1; 2008.

13. Oliveira M, Quental C. A prospecção tecnológica como ferramenta de planejamento estratégico para construção do futuro do Instituto Oswaldo Cruz. Rev Eletron de Comum Inf Saúde [Internet]. 2012 Mar. [citado 17 nov 2015]; 6(1): 50-61. Disponível em: http://www.reciis.icict.fiocruz.br/index.php/reciis/ article/view/607/1248.

14. Brasil. Ministério da Saúde [Internet]. Boletim Epidemiológico. 2015 - [citado 4 nov de 2015]. v. 46, n. 21. 2015. Disponível em: http://portalsaude.saude.gov.br/images/pdf/2015/agosto/03/2014-020..pdf

15. Prates MV, Bloch C. Peptídeos antimicrobianos. Biotecnologia Ciência \& Desenvolvimento [Internet]. 2000 Nov-Dez. [citado 4 nov 2015]; 17(3): 30-36. Disponível em: http://www.biotecnologia.com.br/ revista/bio17/17 pa.pdf

16. Faria AF, Suzuki JÁ, Almeida ALT, Rodrigues MFC, Vidigal CC, Ferreira NM, Mendonça PJF. Promoção da interação universidade-empresa: estudo de caso do CenTev/UFV. XV Congresso de Gestão de Tecnologia Latino-Iberoamericano; Porto; 2013.

17. Fundação Oswaldo Cruz [Internet]. História. 2015. [citado 4 nov 2015]. Disponível em: http://www. agencia.fiocruz.br/doen\%C3\%A7a-de-chagas 